•综述・

\title{
植物氮形态利用策略及对外来植物入侵性的影响
}

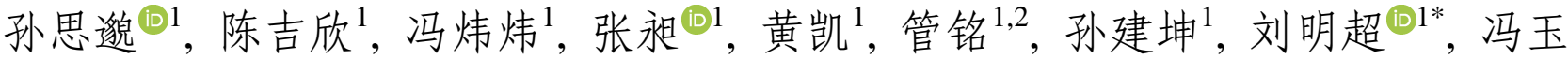 \\ 龙 (iD)
}

1. 辽宁省全球变化与生物入侵重点实验室, 沈阳农业大学生物科学技术学院, 沈阳 110866; 2. 台州学院生命科学学院, 浙江台州 318000 摘要: 氮是影响外来植物入侵性的重要因素之一, 但相关研究多关注土壤氮水平的效应, 较少考虑氮形态的作用。为从土壤 氮形态利用的角度阐释外来植物的入侵机制, 本文在植物氮形态利用策略分析的基础上, 综述了外来植物氮形态利用的偏好 性及其对入侵性的影响。植物的氮形态利用策略有偏好性和可塑性两种, 这可能与植物对土壤氮形态特性的长期适应有关; 植物不仅可以对土壤氮形态做出响应，而且还能改造土壤氮形态，并对改变后的土壤氮形态做出反馈响应。很多外来植物入 侵硝态氮占优势的干扰生境，偏好硝态氮的外来植物与本地植物竞争硝态氮; 而偏好铵态氮的外来植物通过抑制土壤硝化 作用, 营造铵态氮环境, 促进自身生长, 同时抑制偏好硝态氮的本地植物生长。然而，植物氮形态利用策略不是一成不变的， 而是受多种生物和非生物因素共同作用影响的复杂过程, 今后应加强多因素交互作用对外来入侵植物氮形态利用策略的影 响及机制研究，更好地揭示氮形态利用策略，尤其是氮形态利用的可塑性与外来植物入侵性的关系。

关键词: 植物氮形态利用策略; 氮形态偏好; 氮形态可塑性; 土壤氮形态; 适应进化; 外来植物入侵

孙思邀, 陈吉欣, 冯炜炜, 张氹, 黄凯, 管铭, 孙建坤, 刘明超, 冯玉龙 (2021) 植物氮形态利用策略及其对外来植物入侵性的影响. 生物多样性, 29, 72-80. doi: 10.17520/biods.2020072.

Sun SM, Chen JX, Feng WW, Zhang C, Huang K, Guan M, Sun JK, Liu MC, Feng YL (2021) Plant strategies for nitrogen acquisition and their effects on exotic plant invasions. Biodiversity Science, 29, 72-80. doi: 10.17520/biods.2020072.

\section{Plant strategies for nitrogen acquisition and their effects on exotic plant invasions}

Simiao Sun ${ }^{(11}$, Jixin Chen $^{1}$, Weiwei Feng ${ }^{1}$, Chang Zhang ${ }^{(1)}$, Kai Huang ${ }^{1}$, Ming Guan ${ }^{1,2}$, Jiankun Sun ${ }^{1}$, Mingchao Liu $^{\left(\mathbb{D} 1^{*}\right.}$, Yulong Feng ${ }^{\left(\mathbb{D} 1^{*}\right.}$

1 Liaoning Key Laboratory for Biological Invasions and Global Changes, College of BioScience and BioTechnology, Shengyang Agricultural University, Shenyang 110866

2 School of Life Sciences, Taizhou University, Taizhou, Zhejiang 318000

\section{ABSTRACT}

Aim: Available nitrogen is a major factor that influences invasion success of exotic plants. However, our understanding behind the effects of different forms of soil nitrogen on exotic plant invasions remains inconclusive. To elucidate invasion mechanisms of exotic plants in context of soil nitrogen, we reviewed nitrogen form preferences of invasive plants and their effects on invasiveness in comparison to native plant strategies.

Progresses: Different plants have different nitrogen form acquisition strategies, including preference and plasticity, which may be associated with long-term adaption to characteristics of soil nitrogen form and occur at different scales. In addition, some plants can modulate soil nitrogen forms, and respond to altered soil nitrogen. Many invasive plants succeed in disturbed habitats where nitrate is the dominant form of soil nitrogen, as they prefer nitrate and compete for nitrate with co-occurring native plants. Whereas ammonium-preferring invasive plants may accumulate ammonium in soil by inhibiting soil nitrification, thereby facilitating their own growth and inhibiting native plant growth, further contributing to invasion success. However, plant strategies for nitrogen acquisition are not invariable and can be

收稿日期: 2020-03-02; 接受日期: 2020-06-01

基金项目: 国家自然科学基金(31670545; 31971557; 31470575)和国家重点研发计划(2017YFC1200101)

* 共同通讯作者 Co-authors for correspondence. E-mail: fyl@syau.edu.cn; liumingchao8866@163.com. 
influenced by many biotic and abiotic factors and interactions.

Prospects: In future research we should study plant nitrogen acquisition strategies along with their ecological and evolutionary mechanisms in conjunction with ecosystem and environmental factors, especially plasticity of nitrogen form utilization and its relationship with invasiveness of exotic plants. In addition, mycorrhizal fungi may also affect plant utilization of different nitrogen forms, which is also worth studying.

Key words: adaptive evolution; exotic plant invasions; plant nitrogen form preference; plant strategies for acquiring different nitrogen forms; plasticity of nitrogen form utilization; soil nitrogen forms

随着经济全球化与现代化进程的加快, 世界各 地外来入侵植物的种类越来越多, 危害越来越严重 (Ochocki \& Miller, 2017; Seebens et al, 2017)。外来 植物入侵已成为全球性的重大经济、环境和科学问 题, 给人类带来了严峻的挑战。因此, 迫切需要揭 示外来植物的入侵机制, 这不仅有利于对外来植物 进行风险评估, 也是有效防控外来植物入侵的前提 (Catford et al, 2009; Lau \& Schultheis, 2015)。氮素是 植物生长发育的必需元素之一, 土壤氮有效性的提 高能促进外来植物入侵(王满莲和冯玉龙, 2005; Lee et al, 2012; Castro-Díez et al, 2014)。外来入侵植物不 仅对土壤氮的吸收能力强, 具有更高的叶片氮含量 (Huang et al, 2020), 而且还能更有效地利用叶片中 的氮(Feng et al, 2009, 2011)。但是, 相关研究大多只 考虑氮水平的影响, 较少考虑氮形态的效应。

在大多数生态系统中, 植物主要通过细根和与 之共生的菌根真菌吸收利用土壤中的 $\mathrm{NH}_{4}^{+} 、 \mathrm{NO}_{3}^{-}$及 小分子有机氮, 但不同植物对不同形态氮的吸收利 用能力不同, 且土壤氮形态具有时空异质性(Lee et al, 2012; Shannon-Firestone et al, 2015; Kong et al, 2019; Gao et al, 2020)。自然条件下, 一些植物总是 偏好吸收利用土壤中某一特定形态的氮, 且这种偏 好利用策略不受土壤中不同形态氮比例的影响 (Huangfu et al, 2016; Chen \& Chen, 2019); 而另一些 植物却总是吸收利用土壤中的优势氮形态, 表现出 氮形态利用的可塑性(Houlton et al, 2007; Wang \& Macko, 2011; Andersen \& Turner, 2013; Russo et al, 2013)。外来植物氮形态利用策略的差异必然会影响 其入侵性在氮形态时空异质性生境中的表达, 以及 全球变化背景下其入侵性的演化, 但相关研究较 少。为了从氮形态利用角度理解外来植物的入侵性, 本文首先介绍了植物氮形态利用策略、形成机制及 生态学意义, 在此基础上综述了外来植物氮形态利 用策略及其对入侵性的影响。
1 植物的氮形态利用策略、形成机制及生 意义

在土壤微生物的作用下, 土壤中复杂的含氮有 机物逐步分解为简单的小分子氨基化合物, 再经氨 化作用转化成氨和其他更简单的中间产物, 大部分 氨与有机或无机酸结合形成铵盐, 或被生物吸收利 用, 或在微生物的作用下进一步氧化成硝酸盐。气 候、土壤理化性质及其交互作用均能直接或间接影 响土壤氮转化过程中的关键微生物, 如氨氧化细 菌、氨氧化古菌和亚硝酸氧化菌等(Attard et al, 2010; Ke et al, 2013), 进而影响土壤氮转化过程、土壤氮 形态特性及植物对土壤氮的利用(Dannenmann et al, 2016; Hu et al, 2016)。

\section{1 植物氮形态利用的偏好性}

大量研究表明, 不同植物的氮形态利用策略不 同, 相对于小分子有机氮, 大多数植物明显偏好吸 收利用土壤中的无机氮(Harrison et al, 2007; Ashton et al, 2010; Liu M et al, 2017; Gao et al, 2020)。而且, 不同植物对不同形态无机氮的吸收利用能力也有 差异, 有些植物偏好吸收利用土壤中的 $\mathrm{NO}_{3}^{-}$, 而另 一些植物则偏好利用土壤中的 $\mathrm{NH}_{4}^{+}$(Huangfu et al, 2016; Zhang ZL et al, 2018; Chen \& Chen, 2019)。物 种在群落中的优势度与其氮形态偏好和土壤优势 氮形态密切相关, 优势物种常常利用土壤中最丰富 的氮形态, 而非优势物种则利用土壤中的非优势氮 形态(McKane et al, 2002; Gao et al, 2020)。在自然生 态系统中, 不同植物通过吸收利用不同的土壤氮形 态降低对土壤氮素的竞争, 有利于促进物种共存、 维持生物多样性和生态平衡(Ashton et al, 2010; Song et al, 2015)。

植物对不同氮形态的偏好与其对不同形态氮 的吸收和同化策略有关(Andrews et al, 2013; von Wittgenstein et al, 2014; Coskun et al, 2017)。植物吸 
收 $\mathrm{NH}_{4}^{+}$与 $\mathrm{NO}_{3}^{-}$的机制不同, 前者需要铵转运蛋白, 后者需要硝酸盐转运蛋白(Li et al, 2017; Wang et al, 2018)。植物吸收的 $\mathrm{NH}_{4}^{+}$通过谷氨酰胺合成酶同化为 谷氨酰胺, 再由谷氨酸合成酶催化生成谷氨酸, 进 而合成其他氨基酸和蛋白质供植物利用; 但吸收的 $\mathrm{NO}_{3}^{-}$必须先通过硝酸还原酶还原成 $\mathrm{NH}_{4}^{+}$, 才能经过 上述过程合成氨基酸等(Coskun et al, 2017; Rajsz et $\mathrm{al}, 2019)$ 。在高浓度的 $\mathrm{NH}_{4}^{+}$下, 很多喜 $\mathrm{NO}_{3}^{-}$植物会表 现出中毒症状, 而喜 $\mathrm{NH}_{4}^{+}$植物则可通过调控 $\mathrm{NH}_{4}^{+}-\mathrm{N}$ 吸收或其他解毒机制防止 $\mathrm{NH}_{4}^{+}$中毒 (Mahmood \& Kaiser, 2003; Wang \& Macko, 2011)。

自然界中, 植物偏好的氮形态常与生境中土壤 的主要氮形态一致。例如, 分布在以 $\mathrm{NO}_{3}^{-}$为优势氮 形态的中性或碱性土壤(Zhang JB et al, 2018)、干旱 和半干旱生境中的植物(Ashton et al, 2010; Engel et $\mathrm{al}, 2019)$, 以及低海拔阔叶林、荒地和农田中的植物 (Shannon-Firestone et al, 2015; Zhang et al, 2016)多 偏好 $\mathrm{NO}_{3}^{-}$。分布在以 $\mathrm{NH}_{4}^{+}$为优势氮形态的酸性土壤 (Kronzucker et al, 1997; Wallander et al, 1997; Lee, 1998; Li et al, 2013)、湿润生境(Wang \& Macko, 2011)、高海拔针叶林中的植物(Malagoli et al, 2000; Pyatt et al, 2001; Zhang ZL et al, 2018)多偏好 $\mathrm{NH}_{4}^{+}$。 分布在 $\mathrm{NH}_{4}^{+}$和有机氮占优势的极地和高山生境中的 植物多偏好 $\mathrm{NH}_{4}^{+}$和有机氮(Averill \& Finzi, 2011; Shan et al, 2014)。而长期生长在小分子有机氮占优 势的北极苔原和北方森林(boreal forests)中的植物 多偏好小分子有机氮(McKane et al, 2002; Zhang ZL et al, 2018)。上述研究结果暗示, 植物对不同形态氮 的偏好可能与其对土壤中主要氮形态的长期适应 有关, 长期生长在 $\mathrm{NO}_{3}^{-} 、 \mathrm{NH}_{4}^{+}$或小分子有机氮占优 势的土壤中, 植物可能进化出相应的氮形态偏好利 用策略。

植物不仅可以对大范围区域环境中的土壤氮 形态做出响应, 也能对不同尺度的局域环境中的氮 形态(空间异质性)做出响应, 而且同种植物也能通 过适应不同的土壤氮形态而发生遗传分化。例如, 在干旱草原, 浅根系的禾本科牧草一般偏好吸收利 用 $\mathrm{NH}_{4}^{+}$, 而深根系的灌木偏好 $\mathrm{NO}_{3}^{-}$, 两类植物偏好 的氮形态与其根系所处的土壤分层中的主要氮形 态一致(Gherardi et al, 2013)。Wang和Macko (2011) 研究发现, 从干旱到湿润生境, 土壤优势氮形态由 $\mathrm{NO}_{3}^{-}$逐渐转变为 $\mathrm{NH}_{4}^{+}$, 同种植物 (如 Schmidtia pappophoroides)的氮形态偏好也发生相应的转变。 Song等(2015)研究发现, 用不同形态的氮长期处理 后(8年), 具有相同氮偏好的高山植物的氮形态偏好 发生了明显分化, 支持植物氮形态偏好的适应性进 化机制。

植物不仅可以适应, 而且还能调控土壤氮形态, 并对改变后的土壤氮形态做出反馈响应。很多植物 都能通过根系分泌物、地上和地下调落物及地上淋 溶物影响土壤氮素转化关键微生物的群落结构与 功能, 进而影响土壤氮转化过程和氮形态(Chen et al, 2009; Subbarao et al, 2012; Tharayil et al, 2013; Diallo et al, 2015)。这类植物可以在其根际、冠层下 形成有别于其周围本底环境的土壤氮形态; 如果连 续成片分布, 这类植物还可以形成土壤氮形态不同 于其周围本底环境的不同尺度的斑块。这类植物对 土壤氮形态的改变可能会反过来影响其自身的氮 形态偏好。例如, 金花柚木(Eperua falcata)根系分泌 4',7-二羟基黄烷酮和4,2',4-三差基查耳酮, 降低根 际土壤反硝化细菌丰度, 抑制土壤的反硝化作用, 使土壤积累 $\mathrm{NO}_{3}^{-}$, 自身偏好 $\mathrm{NO}_{3}^{-}$(Michalet et al, 2013)。水稻根系分泌1,9-癸二醇, 抑制氨氧化过程 中的关键酶氨单加氧酶活性, 降低土壤硝化速率, 提高根际土 $\mathrm{NH}_{4}^{+}$含量, 自身偏好 $\mathrm{NH}_{4}^{+}$(Sun et al, 2016; Li et al, 2017)。

菌根真菌在植物氮形态偏好利用中也可能发 挥重要作用。自然界中, 植物根系常与菌根真菌形 成共生体(菌根), 以提高宿主植物对土壤氮等元素 的吸收利用(Kong et al, 2019)。有研究表明, 菌根真 菌对很多植物的氮贡献率可达到 $50 \%$, 甚至更高 (Toussaint et al, 2004; Jin et al, 2005; Tanaka \& Yano, 2005; Mayor et al, 2015)。Seck-Mbengue等(2017)发 现, 在 $\mathrm{NH}_{4}^{+}$占优势的土壤中, 从枝菌根真菌对无芒 虎尾草(Chloris gayana)的氮贡献率是在 $\mathrm{NO}_{3}^{-}$占优势 的土壤中的两倍, 表明其菌根真菌偏好 $\mathrm{NH}_{4}^{+}$。豇豆 (Vigna unguiculata) 的丛枝菌根真菌根内球囊霉 (Glomus intraradices)也偏好吸收 $\mathrm{NH}_{4}^{+}$(Ngwene et al, 2013)。

\section{2 植物氮形态利用的可塑性}

与土壤氮含量类似, 土壤氮形态也具有空间上 的异质性和时间上的波动性(Wilson et al, 2005; Zhang ZL et al, 2018)。在长期的适应进化过程中, 一些植物形成了氮形态利用的可塑性, 即根据土壤 
氮形态特点调整自身氮形态利用策略, 始终利用土 壤优势氮形态 (Tylova-Munzarova et al, 2005; Houlton et al, 2007; Wang et al, 2016)。与具有严格氮 形态偏好的植物相比, 具有氮形态利用可塑性的植 物可以始终利用环境中的主要氮源, 能更好地适应 氮形态时空变化较频繁的异质生境(Andersen \& Turner; 2013)。

Houlton等(2007)研究发现, 随着降水量的逐渐 增加, 夏威夷热带雨林土壤优势氮形态由 $\mathrm{NO}_{3}^{-}$逐渐 转变为 $\mathrm{NH}_{4}^{+}$, 相同功能群的植物对不同氮形态的利 用也发生了相应的改变, 表现出对土壤氮形态利用 的可塑性, 以降低氮获取成本。槐叶苹(Salvinia natans) 是偏 $\mathrm{NH}_{4}^{+}$植物, 自然条件下 $\mathrm{NH}_{4}^{+}$是其最佳氮 源, 但当环境中 $\mathrm{NH}_{4}^{+}$浓度很低且 $\mathrm{NO}_{3}^{-}$浓度很高时, 它会通过吸收 $\mathrm{NO}_{3}^{-}$获取氮素(Jampeetong \& Brix, 2009)。在激烈竞争或胁迫环境下, 群落中的优势植 物通常主要利用土壤中的优势氮形态, 而不是它们 偏好的氮形态, 表现出氮形态利用的可塑性 (McKane et al, 2002)。例如, 在缺乏竞争时, 美国科 罗拉多州干旱高寒草甸优势种偏好 $\mathrm{NO}_{3}^{-}$, 但与非优 势种竞争时, 它们增加了对 $\mathrm{NH}_{4}^{+}$的吸收量(Ashton et al, 2010)。

在我国青藏高原东部, 随着林龄增加(20-70 年), 云杉(Picea asperata)林土壤主要无机氮形态由 $\mathrm{NO}_{3}^{-}$逐渐转变为 $\mathrm{NH}_{4}^{+}$, 与此同时, 云杉利用的主要 无机氮形态也发生相应的变化: 20和30年生云杉主 要利用 $\mathrm{NO}_{3}^{-}, 40$ 和 50 年生云杉对 $\mathrm{NO}_{3}^{-}$和 $\mathrm{NH}_{4}^{+}$的利用 相似, 70年生云杉主要利用土壤 $\mathrm{NH}_{4}^{+}$, 这既与云杉 氮形态利用的可塑性有关, 也与云杉氮形态偏好有 关(Zhang ZL et al, 2018)。云杉通常偏好 $\mathrm{NH}_{4}^{+}$, 但在 演替早期表现出明显的偏 $\mathrm{NO}_{3}^{-}$特性, 表明其氮形态 偏好不是固定不变的, 会随着发育时期或土壤环境 的变化而变化。有研究表明, 不同的生长发育时期, 植物对不同氮形态的利用策略不同(Marschner et al, 1991; Cui et al, 2017); 在植被演替过程中, 多年生 植物的生理特性以及土壤主要氮形态等均会发生 变化, 这可能会引起植物氮获取策略的改变 (Kronzucker et al, 1997; Beyschlag et al, 2009)。

外来植物的氮形态偏好及对入侵的影响

\section{1 外来植物的氮形态偏好}

与本地植物的情况类似, 不同外来入侵植物的
氮形态利用策略也不同。研究表明, 外来入侵植物 柔枝莠竹(Microstegium vimineum)、裂稃燕麦(Avena barbata)、大麦状雀麦(Bromus hordeaceous)、旱雀 麦(B. tectorum)、反枝苋(Amaranthus retroflexus)、三 叶鬼针草(Bidens pilosa)、五爪金龙(Ipomoea cairica) 和薇甘菊 (Mikania micrantha) 等均偏好 $\mathrm{NO}_{3}^{-}$ (Hawkes et al, 2005; MacKown et al, 2009; Lee et al, 2012; Shannon-Firestone et al, 2015; Wang et al, 2017; Chen \& Chen, 2019)。外来入侵植物的氮形态偏好也 与其对生境中土壤主要氮形态的适应有关。很多外 来入侵植物都具有杂草性, 在原产地和入侵地常分 布在 $\mathrm{NO}_{3}^{-}$占优势的干扰生境(陈伟杪等, 2013), 长期 适应高 $\mathrm{NO}_{3}^{-}$环境使这些外来入侵植物偏好 $\mathrm{NO}_{3}^{-}$。

但是, 也有外来入侵植物偏好土壤 $\mathrm{NH}_{4}^{+}$的报 道。例如, 外来入侵植物须芒草 (Andropogon gayanus)、加拿大一枝黄花(Solidago canadensis)、 南美蟛蜞菊(Wedelia trilobata)、黄顶菊(Flaveria bidentis)、飞机草(Chromolaena odorata)和紫茎泽兰 (Ageratina adenophora) 等均偏好 $\mathrm{NH}_{4}^{+}$(RossiterRachor et al, 2009; 高燕, 2014 ${ }^{\mathbb{1}}$; 陈静瑜等, 2015; 侯玉平等, 2015; Huangfu et al, 2016; Hu et al, 2019; Huangfu \& Li, 2019)。在 $\mathrm{NO}_{3}^{-}$占优势的干扰生境中, 这些入侵植物表现出偏 $\mathrm{NH}_{4}^{+}$特性, 这与它们对入侵 地土壤氮形态的调控作用有关。例如, 须芒草入侵 能促进土壤氨化作用, 抑制土壤硝化作用, 使土壤 积累 $\mathrm{NH}_{4}^{+}$(Rossiter-Rachor et al, 2009); 黄顶菊根围 土氨氧化细菌活性、硝化潜势和 $\mathrm{NO}_{3}^{-}$含量均低于非 入侵土壤(赵晓红等, 2015; 李科利等, 2017); 飞机 草入侵地土壤 $\mathrm{NH}_{4}^{+}$含量是非入侵地的1.43倍( Hu et al, 2019); 紫茎泽兰入侵地 $\mathrm{NH}_{4}^{+}$含量是非入侵地的 1.56-2.10倍(Niu et al, 2007; Hu et al, 2019), 根围土 $\mathrm{NH}_{4}^{+}$含量是非根围土的1.20倍(Zhao et al, 2019)。

\section{2 氮形态偏好对外来植物入侵的影响}

外来植物的氮形态偏好必然会影响其在土壤 氮形态时空异质性环境中的入侵性表达与分布, 也 会影响全球变化背景下其入侵性的演化。很多外来 入侵植物偏好 $\mathrm{NO}_{3}^{-}$, 这与它们易于入侵 $\mathrm{NO}_{3}^{-}$占优势 的干扰生境的现象相一致(陈伟涁等, 2013)。在干 扰 生境, 很多本地植物也偏好 $\mathrm{NO}_{3}^{-}$, 入侵植物和本地 植物不可避免地要竞争土壤中的 $\mathrm{NO}_{3}^{-}$, 外来植物对

(1) 高燕 (2014) 氮营养对加拿大一枝黄花入侵性的影响. 硕士学位论 文, 中国科学院植物研究所, 北京. 
土壤 $\mathrm{NO}_{3}^{-}$的吸收能力越强, 其在 $\mathrm{NO}_{3}^{-}$占优势的生境 中的入侵性也可能就越强。研究表明, 偏好 $\mathrm{NO}_{3}^{-}$的 外来入侵植物反枝苋能在叶片中积累较高浓度的 $\mathrm{NO}_{3}^{-}$(高达30\%), 牛食用后会中毒(Casteel et al, 1994; Wang et al, 2017)。偏好 $\mathrm{NO}_{3}^{-}$的外来入侵植物在 体内积累 $\mathrm{NO}_{3}^{-}$, 既能提高天敌防御能力(Casteel et al, 1994), 又能作为渗透调节物质提高其抗旱能力 (Song et al, 2006), 促进其入侵性。与小分子有机渗 透调节物质相比, 植物积累 $\mathrm{NO}_{3}^{-}$成本更低, 且同样 能起到渗透调节作用, 提高抗旱性, 有利于偏 $\mathrm{NO}_{3}^{-}$ 外来植物入侵 $\mathrm{NO}_{3}^{-}$占优势的干旱和半干旱生境。

相反, 偏好 $\mathrm{NH}_{4}^{+}$的外来植物更容易入侵 $\mathrm{NH}_{4}^{+}$占 优势的生境(陈静瑜等, 2015)。偏好 $\mathrm{NH}_{4}^{+}$的植物往往 能抑制土壤氨氧化过程, 降低氨氧化速率, 减少 $\mathrm{NO}_{3}^{-}$的生成, 使土壤积累 $\mathrm{NH}_{4}^{+}$(Sun et al, 2016)。例如, 偏好 $\mathrm{NH}_{4}^{+}$的入侵植物加拿大一枝黄花、黄顶菊、南 美蛙蜞菊、飞机草和紫茎泽兰等均能提高土壤 $\mathrm{NH}_{4}^{+}$ 含量, 降低 $\mathrm{NO}_{3}^{-}$含量(高燕, $2014{ }^{\circledR}$; 陈静瑜等, 2015; 侯玉平等, 2015), 这不仅有利于其自身生长, 同时 还能抑制偏好 $\mathrm{NO}_{3}^{-}$的本地植物的生长, 从而促进其 入侵。与此相应, 这些偏 $\mathrm{NH}_{4}^{+}$的外来入侵植物在我 国经常大面积暴发。

全球变化也能影响土壤氮转化和氮形态, 进而 影响外来植物的入侵性, 其中大气氮沉降的影响最 直接也最明显。从氮含量上看, 大气氮沉降可以提 高土壤氮有效性, 促进外来植物的入侵性(Liu YJ et al, 2017; Payne et al, 2017)。从氮形态上看, 目前大 气沉降氮中 $\mathrm{NH}_{4}^{+}$多于 $\mathrm{NO}_{3}^{-}$(Liu et al, 2013; Zhu et al, 2015; Yu et al, 2019), 有利于偏好 $\mathrm{NH}_{4}^{+}$的外来植物 入侵; 但大气沉降氮中 $\mathrm{NO}_{3}^{-}$的比例逐年上升, 未来 可能有利于偏好 $\mathrm{NO}_{3}^{-}$的外来植物入侵。

理论上, 氮形态利用的可塑性有利于外来植物 入侵氮形态变化较大的时空异质性环境, 但截至目 前, 有关外来植物氮形态利用可塑性的研究还很 少。我们还不清楚外来入侵植物氮形态利用的可塑 性情况以及环境和发育阶段等对其的影响, 不清楚 氮形态利用可塑性对外来植物入侵性的影响。

\section{3 问题与展望}

在长期适应进化过程中形成的氮形态利用策

(1) 高燕 (2014) 氮营养对加拿大一枝黄花入侵性的影响. 硕士学位论 文, 中国科学院植物研究所, 北京.
略能影响外来植物入侵生境的选择、机制及在全球 变化背景下其入侵性的演化。但目前有关外来入侵 植物氮形态利用的研究多是在简单条件下进行的, 考虑的影响因素较少, 难以反映其在生态系统中的 真实情况。为更好地理解氮形态利用策略与外来植 物入侵性的关系, 今后需要在自然环境中或控制条 件下, 深入研究地上/地下生物和非生物因素、植物 自身发育阶段及生理状况等多因素综合作用对外 来入侵植物氮形态利用策略的影响, 利用基因组、 转录组、蛋白质组和代谢组等多组学技术, 揭示外 来入侵植物氮形态利用策略的进化生态学、生理学 和分子生物学机制。

有关外来入侵植物氮形态利用可塑性的研究 很少, 我们还不清楚外来入侵植物氮形态利用可塑 性的存在程度、环境的影响及其与氮形态利用偏好 性和系统发生的关系, 以及氮形态利用可塑性在外 来植物成功入侵中的作用, 这些都亟需研究。表型 可塑性是植物适应环境的重要机制之一, 深入研究 外来入侵植物对土壤氮形态的可塑性响应及机制, 可以更好地理解外来植物的入侵性。

有些外来入侵植物具有生态系统工程师的作 用, 能在不同空间尺度上影响土壤氮转化过程中的 关键微生物, 改变土壤氮形态, 深入研究外来入侵 植物影响土壤氮形态的机制、范围及程度, 有助于 揭示外来植物的入侵机制。

菌根真菌是植物获取氮素的重要途径之一, 不 同植物的菌根真菌种类不同, 不同菌根真菌对氮的 吸收能力和氮形态的利用策略不同, 这些差异可能 会影响外来入侵植物的氮形态利用策略及入侵性, 值得深入研究。

\section{ORCID}

孙思戛 (1D) https://orcid.org/0000-0002-0289-1287 张昶 (1) https://orcid.org/0000-0001-8813-3198 刘明超 (D) https://orcid.org/0000-0003-0047-628x 冯玉龙 (1) https://orcid.org/0000-0003-0243-2280 参考文献

Andersen KM, Turner BL (2013) Preferences or plasticity in nitrogen acquisition by understorey palms in a tropical montane forest. Journal of Ecology, 101, 819-825.

Andrews M, Raven JA, Lea PJ (2013) Do plants need nitrate? The mechanisms by which nitrogen form affects plants. Annals of Applied Biology, 163, 174-199.

Ashton IW, Miller AE, Bowman WD, Suding KN (2010) 
Niche complementarity due to plasticity in resource use: Plant partitioning of chemical $\mathrm{N}$ forms. Ecology, 91, 3252-3260.

Attard E, Poly F, Commeaux C, Laurent F, Terada A, Smets BF, Recous S, Roux XL (2010) Shifts between Nitrospiraand Nitrobacter-like nitrite oxidizers underlie the response of soil potential nitrite oxidation to changes in tillage practices. Environmental Microbiology, 12, 315-326.

Averill C, Finzi A (2011) Increasing plant use of organic nitrogen with elevation is reflected in nitrogen uptake rates and ecosystem $\delta^{15} \mathrm{~N}$. Ecology, 92, 883-891.

Beyschlag W, Hanisch S, Friedrich S, Jentsch A, Werner C (2009) ${ }^{15} \mathrm{~N}$ natural abundance during early and late succession in a middle-European dry acidic grassland. Plant Biology, 11, 713-724.

Casteel SW, Johnson GC, Miller MA, Chudomelka HJ, Cupps DE, Haskins HE, Gosser HS (1994) Amaranthus retroflexus (redroot pigweed) poisoning in cattle. Journal of the American Veterinary Medical Association, 204, 1068-1070.

Castro-Díez P, Godoy O, Alonso A, Gallardo A, Saldaña A (2014) What explains variation in the impacts of exotic plant invasions on the nitrogen cycle? A meta-analysis. Ecology Letters, 17, 1-12.

Catford JA, Jansson R, Nilsson C (2009) Reducing redundancy in invasion ecology by integrating hypotheses into a single theoretical framework. Diversity and Distributions, 15, 22-40.

Chen BM, Peng SL, Ni GY (2009) Effects of the invasive plant Mikania micrantha H. B. K. on soil nitrogen availability through allelopathy in South China. Biological Invasions, 11, 1291-1299.

Chen JY, Gu YR, Tian XS, Li WH (2015) Responses of the invasive plant Wedelia trilobata to $\mathrm{NH}_{4}^{+}-\mathrm{N}$ and $\mathrm{NO}_{3}^{-}-\mathrm{N}$. Journal of South China Normal University (Natural Science Edition), 47(5), 84-90. (in Chinese with English abstract) [陈静瑜, 谷妍蓉, 田兴山, 李伟华 (2015) 入侵植物南美 蟛蜞菊对 $\mathrm{NH}_{4}^{+}-\mathrm{N}$ 和 $\mathrm{NO}_{3}^{-}-\mathrm{N}$ 的响应. 华南师范大学学报(自 然科学版), 47(5), 84-90.]

Chen WB, Chen BM (2019) Considering the preferences for nitrogen forms by invasive plants: A case study from a hydroponic culture experiment. Weed Research, 59, 49-57.

Chen WB, Su JQ, Peng SL, Chen BM (2013) Responses of several exotic invasive plant to different nitrogen forms in southern China. Chinese Science Paper Online, http://www.paper.edu.cn/researchpaper/content/201308-113. (in Chinese with English abstract) [陈伟森, 苏锦权, 彭少 麟, 陈宝明 (2013) 华南几种外来入侵植物对不同氮源 的响应. 中国科技论文在线, http://www.paper.edu.cn/ researchpaper/content/201308-113.]

Coskun D, Britto DT, Kronzucker HJ (2017) The nitrogen-potassium intersection: Membranes, metabolism, and mechanism. Plant, Cell \& Environment, 40, 2029-2041.

Cui JH, Yu CQ, Qiao N, Xu XL, Tian YQ, Ouyang H (2017)
Plant preference for $\mathrm{NH}_{4}^{+}$versus $\mathrm{NO}_{3}^{-}$at different growth stages in an alpine agroecosystem. Field Crops Research, 201, 192-199.

Dannenmann M, Bimüller C, Gschwendtner S, Leberecht M, Tejedor J, Bilela S, Gasche R, Hanewinkel M, Baltensweiler A, Kögel-Knabner I, Polle A, Schloter M, Simon J, Rennenberg H (2016) Climate change impairs nitrogen cycling in European beech forests. PLoS ONE, 11, e0158823.

Diallo MD, Guisse A, Sall SN, Dick RP, Assigbetse KB, Dieng AL, Chotte J (2015) Influence of tropical leaf litter on nitrogen mineralization and community structure of ammonia-oxidizing bacteria. Biotechnology, Agronomy, Society and Environnement, 19, 173-183.

Engel RE, Romero CM, Carr P, Torrion JA (2019) Performance of nitrate compared with urea fertilizer in a semiarid climate of the northern Great Plains. Canadian Journal of Soil Science, 99, 345-355.

Feng YL, Lei YB, Wang RF, Callaway RM, Valiente-Banuet A, Inderjit, Li YP, Zheng YL (2009) Evolutionary tradeoffs for nitrogen allocation to photosynthesis versus cell walls in an invasive plant. Proceedings of the National Academy of Sciences, USA, 106, 1853-1856.

Feng YL, Li YP, Wang RF, Callaway RM, Valiente-Banuet A, Inderjit (2011) A quicker return energy-use strategy by populations of a subtropical invader in the non-native range: A potential mechanism for the evolution of increased competitive ability. Journal of Ecology, 99, 1116-1123.

Gao L, Cui XY, Hill, PW, Guo YF (2020) Uptake of various nitrogen forms by co-existing plant species in temperate and cold-temperate forests in northeast China. Applied Soil Ecology, 147, 103398.

Gherardi LA, Sala OE, Yahdjian L (2013) Preference for different inorganic nitrogen forms among plant functional types and species of the Patagonian steppe. Oecologia, 173, 1075-1081.

Harrison KA, Bol R, Bardgett RD (2007) Preferences for different nitrogen forms by coexisting plant species and soil microbes. Ecology, 88, 989-999.

Hawkes CV, Wren IF, Herman DJ, Firestone MK (2005) Plant invasion alters nitrogen cycling by modifying the soil nitrifying community. Ecology Letters, 8, 976-985.

Hou YP, Liu L, Chu H, Ma SJ, Zhao D, Liang RR (2015) Effects of exotic plant Rhus typhina invasion on soil properties in different forest types. Acta Ecologica Sinica, 35, 5324-5330. (in Chinese with English abstract) [侯玉平, 柳林, 初航, 马淑杰, 赵丹, 梁荣荣 (2015) 外来植物火 炬树(Rhus typhina L.)入侵对不同林型土壤性质的影响. 生态学报, 35, 5324-5330.]

Houlton BZ, Sigman DM, Schuur EA, Hedin LO (2007) A climate-driven switch in plant nitrogen acquisition within tropical forest communities. Proceedings of the National Academy of Sciences, USA, 104, 8902-8906. 
Hu CC, Lei YB, Tan YH, Sun XC, Xu H, Liu CQ, Liu XY (2019) Plant nitrogen and phosphorus utilization under invasive pressure in a montane ecosystem of tropical China. Journal of Ecology, 107, 372-386.

Hu HW, Macdonald CA, Trivedi P, Anderson IC, Zheng Y, Holmes B, Bodrossy L, Wang JT, He JZ, Singh BK (2016) Effects of climate warming and elevated $\mathrm{CO}_{2}$ on autotrophic nitrification and nitrifiers in dryland ecosystems. Soil Biology and Biochemistry, 92, 1-15.

Huang K, Kong DL, Lu XR, Feng WW, Liu MC, Feng YL (2020) Lesser leaf herbivore damage and structural defense and greater nutrient concentrations for invasive alien plants: Evidence from 47 pairs of invasive and non-invasive plants. Science of the Total Environment, 723, 137829.

Huangfu CH, Li HY, Chen XW, Liu HM, Wang H, Yang DL (2016) Response of an invasive plant, Flaveria bidentis, to nitrogen addition: A test of form-preference uptake. Biological Invasions, 18, 3365-3380.

Huangfu CH, Li KL (2019) Growing density interacts with competitor identity to modulate nitrogen form preference of an invasive plant. Ecological Indicators, 107, 105641.

Jampeetong A, Brix H (2009) Nitrogen nutrition of Salvinia natans: Effects of inorganic nitrogen form on growth, morphology, nitrate reductase activity and uptake kinetics of ammonium and nitrate. Aquatic Botany, 90, 67-73.

Jin H, Pfeffer PE, Douds DD, Piotrowski E, Lammers PJ, Shachar-Hill Y (2005) The uptake, metabolism, transport and transfer of nitrogen in an arbuscular mycorrhizal symbiosis. New Phytologist, 168, 687-696.

Ke XB, Angel R, Lu YH, Conrad R (2013) Niche differentiation of ammonia oxidizers and nitrite oxidizers in rice paddy soil. Environmental Microbiology, 15, 2275-2292.

Kong DL, Wang JJ, Wu HF, Valverde-Barrantes OJ, Wang RL, Zeng H, Kardol P, Zhang HY, Feng YL (2019) Nonlinearity of root trait relationships and the root economics spectrum. Nature Communications, 10, 2203.

Kronzucker HJ, Yaeesh Siddiqi M, Glass ADM (1997) Conifer root discrimination against soil nitrate and the ecology of forest succession. Nature, 385, 59-61.

Lau JA, Schultheis EH (2015) When two invasion hypotheses are better than one. New Phytologist, 205, 958-960.

Lee JA (1998) The calcicole-calcifuge problem revisited. Advances in Botanical Research, 29, 1-30.

Lee MR, Flory SL, Phillips RP (2012) Positive feedbacks to growth of an invasive grass through alteration of nitrogen cycling. Oecologia, 170, 457-465.

Li H, Hu B, Chu CC (2017) Nitrogen use efficiency in crops: Lessons from Arabidopsis and rice. Journal of Experimental Botany, 68, 2477-2488.

Li SX, Wang ZH, Stewart BA (2013) Responses of crop plants to ammonium and nitrate N. Advances in Agronomy, 118, 205-397.
Li KL, Zhao XH, Liu HM, Yang DL, Qu B, Huangfu CH (2017) Effects of Flaveria bidentis invasion on diversity of soil ammonia-oxidizing archaea. Soils, 49, 1053-1057. (in Chinese with English abstract) [李科利, 赵晓红, 刘红梅, 杨殿林, 曲波, 皇甫超河 (2017) 黄顶菊入侵对土壤氨氧 化古菌群落多样性的影响. 土壤, 49, 1053-1057.]

Liu M, Li CC, Xu XL, Wanek W, Jiang N, Wang HM, Yang XD (2017) Organic and inorganic nitrogen uptake by 21 dominant tree species in temperate and tropical forests. Tree Physiology, 37, 1515-1526.

Liu XJ, Zhang Y, Han WX, Tang AH, Shen JL, Cui ZL, Vitousek P, Erisman, JW, Goulding K, Christie P, Fangmeier A, Zhang F (2013) Enhanced nitrogen deposition over China. Nature, 494, 459-462.

Liu YJ, Oduor AMO, Zhang Z, Manea A, Tooth IM, Leishman MR, Xu XL, van Kleunen M (2017) Do invasive alien plants benefit more from global environmental change than native plants? Global Change Biology, 23, 3363-3370.

Mackown CT, Jones TA, Johnson DA, Monaco TA, Redinbaugh MG (2009) Nitrogen uptake by perennial and invasive annual grass seedlings: Nitrogen form effects. Soil Science Society of America Journal, 73, 1864-1870.

Mahmood T, Kaiser WM (2003) Growth and solute composition of the salt-tolerant kallar grass [Leptochloa fusca (L.) Kunth] as affected by nitrogen source. Plant and Soil, 252, 359-366.

Malagoli M, Dal Canal A, Quaggiotti S, Pegoraro P, Bottacin A (2000) Differences in nitrate and ammonium uptake between Scots pine and European larch. Plant and Soil, 221, 1-3.

Marschner H, Häussling M, George E (1991) Ammonium and nitrate uptake rates and rhizosphere $\mathrm{pH}$ in non-mycorrhizal roots of Norway spruce [Picea abies (L.) karst.]. Trees, 5, 14-21.

Mayor J, Bahram M, Henkel T, Buegger F, Pritsch K, Tedersoo L (2015) Ectomycorrhizal impacts on plant nitrogen nutrition: Emerging isotopic patterns, latitudinal variation and hidden mechanisms. Ecology Letters, 18, 96-107.

McKane RB, Johnson LC, Shaver GR, Nadelhoffer KJ, Rastetter EB, Fry B, Giblin AE, Kielland K, Kwiatkowski BL, Laundre JA, Murray G (2002) Resource-based niches provide a basis for plant species diversity and dominance in arctic tundra. Nature, 415, 68-71.

Michalet S, Rohr J, Warshan D, Bardon C, Roggy JC, Domenach AM, Czarnes S, Pommier T, Combourieu B, Guillaumaud N, Bellvert F, Comte G, Poly F (2013) Phytochemical analysis of mature tree root exudates in situ and their role in shaping soil microbial communities in relation to tree $\mathrm{N}$-acquisition strategy. Plant Physiology and Biochemistry, 72, 169-177.

Ngwene B, Gabriel E, George E (2013) Influence of different mineral nitrogen sources $\left(\mathrm{NO}_{3}^{-}-\mathrm{N}\right.$ vs. $\left.\mathrm{NH}_{4}^{+}-\mathrm{N}\right)$ on arbuscular mycorrhiza development and $\mathrm{N}$ transfer in a Glomus 
intraradices-cowpea symbiosis. Mycorrhiza, 23, 107-117.

Niu HB, Liu WX, Wan FH, Liu B (2007) An invasive aster (Ageratina adenophora) invades and dominates forest understories in China: Altered soil microbial communities facilitate the invader and inhibit natives. Plant and Soil, 294, 73-85.

Ochocki BM, Miller TEX (2017) Rapid evolution of dispersal ability makes biological invasions faster and more variable. Nature Communications, 8, 14315.

Payne RJ, Dise NB, Field CD, Dore AJ, Caporn SJ, Stevens CJ (2017) Nitrogen deposition and plant biodiversity: Past, present, and future. Frontiers in Ecology and the Environment, 15, 431-436.

Pyatt G, Ray D, Fletcher J (2001) An Ecological Site Classification for Forestry in Great Britain. Forestry Commission, Edinburgh.

Rajsz A, Wojtuń B, Mróz L, Żołnierz L, Kempers AJ (2019) Nitrate reductase activity in high-mountain plants: A test across species, growth form and habitat type. Journal of Plant Ecology, 12, 519-530.

Rossiter-Rachor NA, Setterfield SA, Douglas MM, Hutley LB, Cook GD, Schmidt S (2009) Invasive Andropogon gayanus (gamba grass) is an ecosystem transformer of nitrogen relations in Australian savanna. Ecological Applications, 19, 1546-1560.

Russo SE, Kochsiek A, Olney J, Thompson L, Miller AE, Tan S (2013) Nitrogen uptake strategies of edaphically specialized Bornean tree species. Plant Ecology, 214, 1405-1416.

Seck-Mbengue MF, Müller A, Ngwene B, Neumann E, George E (2017) Transport of nitrogen and zinc to rhodes grass by arbuscular mycorrhiza and roots as affected by different nitrogen sources $\left(\mathrm{NH}_{4}^{+}-\mathrm{N}\right.$ and $\left.\mathrm{NO}_{3}^{-}-\mathrm{N}\right)$. Symbiosis, 73, 191-200.

Seebens H, Blackburn TM, Dyer EE, Genovesi P, Hulme PE, Jeschke JM, Pagad S, Pyšek P, Winter M, Arianoutsou M, Bacher S, Blasius B, Brundu G, Capinha C, Celesti-Grapow L, Dawson W, Dullinger S, Fuentes N, Jäger H, Kartesz J, Kenis M, Kreft H, Kühn I, Lenzner B, Liebhold A, Mosena A, Moser D, Nishino M, Pearman D, Pergl J, Rabitsch W, Rojas-Sandoval J, Roques A, Rorke S, Rossinelli S, Roy HE, Scalera R, Schindler S, Štajerová K, Tokarska-Guzik B, van Kleunen M, Walker K, Weigelt P, Yamanaka T, Essl F (2017) No saturation in the accumulation of alien species worldwide. Nature Communications, 8, 14435.

Shan S, Coleman M, Kimsey M (2014) Soil soluble nitrogen availability across an elevation gradient in a cold-temperate forest ecosystem. Soil Science Society of America Journal, 78, 217-224.

Shannon-Firestone S, Reynolds HL, Phillips RP, Flory SL, Yannarell A (2015) The role of ammonium oxidizing communities in mediating effects of an invasive plant on soil nitrification. Soil Biology and Biochemistry, 90,
266-274.

Song J, Ding XD, Feng G, Zhang FS (2006) Nutritional and osmotic roles of nitrate in a euhalophyte and a xerophyte in saline conditions. New Phytologist, 171, 357-366.

Song MH, Zheng LL, Suding KN, Yin TF, Yu FH (2015) Plasticity in nitrogen form uptake and preference in response to long-term nitrogen fertilization. Plant and Soil, 394, 215-224.

Subbarao GV, Sahrawat KL, Nakahara K, Ishikawa T, Kishii M, Rao IM, Hash CT, George TS, Srinivasa Rao P, Nardi P, Bonnett D, Berry W, Suenaga K, Lata JC (2012) Biological nitrification inhibition-A novel strategy to regulate nitrification in agricultural systems. Advances in Agronomy, 114, 249-302.

Sun L, Lu YF, Yu FW, Kronzucker HJ, Shi WM (2016) Biological nitrification inhibition by rice root exudates and its relationship with nitrogen-use efficiency. New Phytologist, 212, 646-656.

Tanaka Y, Yano K (2005) Nitrogen delivery to maize via mycorrhizal hyphae depends on the form of $\mathrm{N}$ supplied. Plant, Cell \& Environment, 28, 1247-1254.

Tharayil N, Alpert P, Bhowmik P, Gerard P (2013) Phenolic inputs by invasive species could impart seasonal variations in nitrogen pools in the introduced soils: A case study with Polygonum cuspidatum. Soil Biology and Biochemistry, 57, 858-867.

Toussaint JP, St-Arnaud M, Charest C (2004) Nitrogen transfer and assimilation between the arbuscular mycorrhizal fungus Glomus intraradices Schenck \& Smith and Ri T-DNA roots of Daucus carota L. in an in vitro compartmented system. Canadian Journal of Microbiology, 50, 251-260.

Tylova-Munzarova E, Lorenzen B, Brix H, Votrubova O, (2005) The effects of $\mathrm{NH}_{4}^{+}$and $\mathrm{NO}_{3}^{-}$on growth, resource allocation and nitrogen uptake kinetics of Phragmites australis and Glyceria maxima. Aquatic Botany, 81, 326-342.

von Wittgenstein NJJB, Le CH, Hawkins BJ, Ehlting J (2014) Evolutionary classification of ammonium, nitrate, and peptide transporters in land plants. BMC Evolutionary Biology, 14, 1-17.

Wallander H, Arnebrant K, Östrand F, Kårén O (1997) Uptake of ${ }^{15} \mathrm{~N}$-labelled alanine, ammonium and nitrate in Pinus sylvestris L. ectomycorrhiza growing in forest soil treated with nitrogen, sulphur or lime. Plant and Soil, 195, 329-338.

Wang CY, Zhou JW, Liu J, Jiang K (2017) Differences in functional traits between invasive and native Amaranthus species under different forms of $\mathrm{N}$ deposition. The Science of Nature, 104, 59.

Wang LX, Macko SA (2011) Constrained preferences in nitrogen uptake across plant species and environments. Plant, Cell \& Environment, 34, 525-534.

Wang ML, Feng YL (2005) Effects of soil nitrogen levels on morphology, biomass allocation and photosynthesis in 
Ageratina adenophora and Chromolaena odorata. Acta Phytoecologica Sinica, 29, 697-705. (in Chinese with English abstract) [王满莲, 冯玉龙 (2005) 紫茎泽兰和飞 机草的形态、生物量分配和光合特性对氮营养的响应. 植 物生态学报, 29, 697-705.]

Wang RX, Tian YQ, Ouyang SN, Xu XL, Xu FZ, Zhang Y (2016) Nitrogen acquisition strategies used by Leymus chinensis and Stipa grandis in temperate steppes. Biology and Fertility of Soils, 52, 951-961.

Wang YY, Cheng YH, Chen KE, Tsay YF (2018) Nitrate transport, signaling, and use efficiency. Annual Review of Plant Biology, 69, 85-122.

Wilson SM, Pyatt DG, Ray D, Malcolm DC, Connolly T (2005) Indices of soil nitrogen availability for an ecological site classification of British forests. Forest Ecology and Management, 220, 51-65.

Yu GR, Jia YL, He NP, Zhu JX, Chen Z, Wang QF, Piao SL, Liu XJ, He HL, Guo XB, Wen Z, Li P, Ding GA, Goulding K (2019) Stabilization of atmospheric nitrogen deposition in China over the past decade. Nature Geoscience, 12, 424-429.

Zhang JB, Wang J, Müller C, Cai ZC (2016) Ecological and practical significances of crop species preferential $\mathrm{N}$ uptake matching with soil $\mathrm{N}$ dynamics. Soil Biology and Biochemistry, 103, 63-70.

Zhang JB, Cai ZC, Müller C (2018) Terrestrial N cycling associated with climate and plant-specific $\mathrm{N}$ preferences: A review. European Journal of Soil Science, 69, 488-501.

Zhang ZL, Li N, Xiao J, Zhao CZ, Zou TT, Li DD, Liu Q, Yin HJ (2018) Changes in plant nitrogen acquisition strategies during the restoration of spruce plantations on the eastern Tibetan Plateau, China. Soil Biology and Biochemistry, 119, 50-58.

Zhao MX, Lu XF, Zhao HX, Yang YF, Hale LR, Gao Q, Liu WX, Guo JY, Li Q, Zhou JZ, Wan FH (2019) Ageratina adenophora invasions are associated with microbially mediated differences in biogeochemical cycles. Science of the Total Environment, 677, 47-56.

Zhao XH, Yang DL, Wang H, Liu HM, Qu B, Huangfu CH (2015) Effects of Flaveria bidentis invasion on soil nitrogen cycling and soil microbial biomass in different regions. Acta Prataculturae Sinica, 24(2), 62-69. (in Chinese with English abstract) [赵晓红, 杨殿林, 王慧, 刘红梅, 曲波, 皇甫超 河 (2015) 黄顶菊入侵对不同地区土壤氮循环及微生物 量的影响. 草业学报, 24(2), 62-69.]

Zhu JX, He NP, Wang QF, Yuan GF, Wen D, Yu GR, Jia YL (2015) The composition, spatial patterns, and influencing factors of atmospheric wet nitrogen deposition in Chinese terrestrial ecosystems. Science of the Total Environment, 511, 777-785. 